\title{
Tratamento nutricional para gestante com DMG na região Amazônica: revisão
}

\section{bibliográfica}

\author{
Nutritional treatment for pregnant women with GDM in the Amazon region: literature review \\ Tratamiento nutricional para mujeres embarazadas con DMG en la región amazónica: revisión de \\ la literatura
}

\author{
Andrielly Sales dos Santos \\ ORCID: https://orcid.org/0000-0003-3302-9312 \\ Centro Universitário Fametro, Brasil \\ E-mail: andriellysales21@gmail.com \\ Celiane da Costa Gomes \\ ORCID: https://orcid.org/0000-0002-4665-9949 \\ Centro Universitário Fametro, Brasil \\ E-mail: selianne.costa@gmail.com \\ Kamilla Pinheiro Motta \\ ORCID: https://orcid.org/0000-0003-0434-4292 \\ Centro Universitário Fametro, Brasil \\ E-mail: kamilapinheiro94@gmail.com \\ José Carlos de Sales Ferreira \\ ORCID: https://orcid.org/0000-0002-1867-8229 \\ Centro Universitário Fametro, Brasil \\ E-mail: jcarlos.sales@gmail.com \\ Rebeca Sakamoto Figueiredo \\ ORCID: https://orcid.org/0000-0002-9819-8099 \\ Centro Universitário Fametro, Brasil \\ E-mail: rebeca.figueiredo@fametro.edu.br
}

\begin{abstract}
Resumo
Introdução: Diabetes Mellitus Gestacional, é definida pela presença de intolerância à glicose no período gravídico e outros tipos específicos. Entretanto, ressalta-se ainda a existência de duas classes, o chamado pré-diabetes e a tolerância à glicose diminuída. Esses dois últimos tipos não são caracterizados como formas clínicas, mas são fatores indutores para o progresso da patologia. Objetivo Geral: Este estudo tem como objetivo discutir e esclarecer sobre o Diabetes Mellitus Gestacional e as possíveis intervenções, cuidados e complicações voltada da região norte, mas especificado no estado do Amazonas. Metodologia: Trata-se de uma revisão integrativa da literatura que objetivou identificar os cuidados do tratamento nutricional e a importância do acompanhamento do pré-Natal corretamente. Resultado e Discussão: A importância da terapia nutricional nessas pacientes, com enfoque nos alimentos da região amazônica que ajudam a controlar e reduzir a glicemia sanguínea. Conclusão: Através da literatura, foi notável a importância da dieta, exercício físico e controle da glicemia durante o tratamento, uma vez que os dados sobre tratamento medicamentoso ainda são insuficientes e controversos.
\end{abstract}

Palavras-chave: Diabetes gestacional; Diabetes Mellitus; Nutrição; Terapia.

\begin{abstract}
Introduction: Gestational Diabetes Mellitus, defined by the presence of glucose intolerance during pregnancy and other specific types. However, the existence of two classes is also highlighted, the so-called pre-diabetes and impaired glucose tolerance. These last two types are not characterized as clinical forms, but are inducing factors for the progress of the pathology. General Objective: This study aims to discuss and clarify about Gestational Diabetes Mellitus and as possible, care and complications focused on the northern region, but specified in the state of Amazonas. Methodology: This is an integrative literature review that aims to identify care for nutritional treatment and the importance of monitoring prenatal care correctly. Results and Discussion: The importance of nutritional therapy in patients, focusing on foods from the Amazon region that prevent and reduce blood glucose. Conclusion: Through the literature, the importance of diet, exercise and blood glucose control during treatment was remarkable, since data on drug treatment are still insufficient and controversial.
\end{abstract}

Keywords: Gestational diabetes; Diabetes Mellitus; Nutrition; Nutritional therapy. 


\begin{abstract}
Resumen
Introducción: Diabetes Mellitus gestacional, definida por la presencia de intolerancia a la glucosa durante el embarazo y otros tipos específicos. Sin embargo, también se destaca la existencia de dos clases, la denominada prediabetes y la intolerancia a la glucosa. Estos dos últimos tipos no se caracterizan como formas clínicas, sino que son factores inductores del progreso de la patología. Objetivo general: Este estudio tiene como objetivo discutir y esclarecer sobre la Diabetes Mellitus Gestacional y en lo posible, la atención y las complicaciones enfocadas en la región norte, pero especificadas en el estado de Amazonas. Metodología: Se trata de una revisión integradora de la literatura que tiene como objetivo identificar los cuidados para el tratamiento nutricional y la importancia de monitorear correctamente la atención prenatal. Resultados y Discusión: La importancia de la terapia nutricional en pacientes, enfocándose en alimentos de la región amazónica que previenen y reducen la glucemia. Conclusión: A través de la literatura, la importancia de la dieta, el ejercicio y el control de la glucemia durante el tratamiento fue notable, ya que los datos sobre el tratamiento farmacológico son aún insuficientes y controvertidos.
\end{abstract}

Palabras clave: Diabetes gestacional; Diabetes mellitus; Nutrición; Terapia nutricional.

\title{
1. Introdução
}

Diabetes Mellitus Gestacional, é definida pela presença de intolerância à glicose no período gravídico e outros tipos específicos. Entretanto, ressalta-se ainda a existência de duas classes, o chamado pré-diabetes e a tolerância à glicose diminuída. Esses dois últimos tipos não são caracterizados como formas clínicas, mas são fatores indutores para o progresso da patologia (ADA, 2015). A DMG é caracterizada pela intolerância a carboidratos diagnosticada pela primeira vez no período da gravidez, podendo ou não persistir após o parto. Esse distúrbio metabólico tem prevalência global estimada entre 1 e $14 \%$ e a média brasileira é de, aproximadamente, 7\% (Hunt, 2008; Packer, 2016; Battarbee et al., 2020).

O DMG acarreta inúmeras complicações tanto para a mãe quanto para o feto. A gestante diabética possui o risco de apresentar cetoacidose, lesões vasculares e neuropatia. Podem ocorrer hiperglicemia e consequente choque, resultante das 17 variações da secreção de insulina. Também ocorre maior risco de desenvolver infecções graves e recorrentes, hipertensão, deslocamento prematuro da placenta e abortamento espontâneo. Durante o parto, pode haver distocia e lesões vaginais, devido a macrossomia fetal, hemorragia puerperal e morte. No lactente pode ocorrer imaturidade pulmonar e síndrome da angústia respiratória; traumatismos obstétricos múltiplos associados a macrossomia e ao parto vaginal difícil; anomalias congênitas; hiperglicemia, hipocalcemia e hiper bilirrubinemia; predisposição ao diabetes e morte (Santos et al., 2012).

Essa alteração metabólica pode causar diversas complicações tanto para as gestantes, quanto para os fetos. Nas gestantes, aumentam-se os riscos de desenvolverem pré-eclâmpsia, diabetes tipo II, doenças cardiovasculares e síndrome metabólica, e podem levar ao parto prematuro e cesárea. Já no caso dos fetos, elevam-se as chances de aparecimento de malformações congênitas nos sistemas esquelético, cardíaco e neurológico, além de poderem manifestar a macrossomia e hiper bilirrubinemia e, junto a isso, cresce a probabilidade de serem diabéticos tipo II na fase adulta (Galerneau \& Inzucchi, 2004; Reece, 2010; Mohsin et al., 2016).

\section{Metodologia}

O presente estudo efetivou-se através de pesquisa descritiva, na qual propôs a identificação, registro e análise das características, fatores ou variáveis que se relacionam com o fenômeno ou processo (Perovano, 2014). Dessa forma, embasada em referências bibliográficas e científicas, relatando complicações DMG na hora do parto das gestações.

Para extrair os dados dos artigos selecionados, será necessária a utilização de um instrumento previamente elaborado capaz de assegurar que a totalidade dos dados relevantes seja extraída, minimizar o risco de erros na transcrição, garantir precisão na checagem das informações e servir como registro (Souza, Silva \& Carvalho, 2010)

Para elaboração da pesquisa, foram realizados levantamentos bibliográficos fazendo o uso de materiais publicados em forma de revistas, órgãos oficiais, artigos em sites como SCIELO (Scientific Eletronic Library), PubMed (Serviço da National 
Library of Medicine) e Biblioteca Virtual de Saúde (BVS). Para a busca dos artigos, utilizaram-se os descritores: Diabetes Gestacional, Diabetes Mellitus, Nutrição, Terapia Nutricional.

Para uma análise crítica e reflexiva dos estudos incluídos na revisão, será realizada uma leitura minuciosa e criteriosa destacando os que atingirem os critérios de inclusão e que contemplarem o objetivo proposto, para viabilizar o resultado da pesquisa de forma clara e objetiva.

Serão incluídos os artigos publicados em língua portuguesa nos últimos onze anos, com texto completo, disponível on-line, com acesso livre. Serão excluídos da amostra os artigos publicados em línguas estrangeiras, os que não apresentarem o texto na integra, artigos que não apresentavam relação direta com o tema, resumos, monografias, dissertações, teses e artigos repetidos.

\section{Resultados e Discussão}

\subsection{Diabetes Mellitus Gestacional}

No Brasil, a prevalência de DMG em mulheres com idade superior a 20 anos, atendidas pelo Sistema Único de Saúde (SUS), foi de 7,6\%4. Esses dados demonstram a relevância do DMG devido tanto a ser uma complicação adquirida durante a curso de gravidez, e por ser um problema de saúde pública. Além disso, o ao contrário do que acontece as mulheres que tem diabetes ante gravidez (DM tipo I ou II), aquelas que descobrem que tem diabetes durante o curso da gravidez atual tem adição de uma condição de risco que vai além da característica especificas inerentes a qualquer baixo risco de gravidez (Ciências Saúde - 22 Sup 1:S93-S100, 2011).

O diabetes mellitus gestacional (DMG) é considerado o distúrbio metabólico mais comum durante a gravidez, e sua prevalência varia de $3 \%$ a $13 \%$ em todas as gestantes, dependendo da população avaliada e dos critérios utilizados (SBD, 2014-2015). A ineficácia do cuidado pré-natal para controlar o DMG aumentará os riscos durante a gravidez, complicações e efeitos adversos nas mães e bebês durante o período pré-natal e neonatal. Pode-se dizer que o DMG aumenta a possibilidade de a mulher sofrer de DM2 após o parto e, a cada gravidez, aumenta também o risco de o bebê sofrer da mesma doença (Nolan; McCrone, Chertok, 2011; Araújo et al., 2013).

Normalmente, o diagnóstico de DMG é feito por meio de busca ativa, teste de provocação e uso de sobrecarga de glicose, no segundo trimestre. Porém, a recomendação atual é realizar o rastreamento precoce das gestantes para DMG no primeiro exame de pré-natal, para que sejam identificados casos de DM pré-existentes que não possam ser considerados DMG (SMCD, 2010; Weinert, 2011; IADPSG Consensus Panel, 2010). Em seu estudo, Simon, Marques e Farhat (2013) apontaram que ainda existem muitas controvérsias sobre o ponto de corte da glicemia de jejum no rastreamento do DMG, e que esse parâmetro varia de acordo com a população e os pontos de redução dos funcionários estudados. Esses autores relataram que nenhum caso de DMG foi encontrado em pacientes com glicemia de jejum abaixo de $90 \mathrm{mg} / \mathrm{dl}$ no primeiro trimestre ou sem fatores de risco.

A SBD recomenda o uso de novos padrões internacionais para o diagnóstico de DMG, pois, apesar de suas limitações, são os únicos que comprovadamente mostram a relação entre os níveis de glicose no sangue materno e seus efeitos na saúde do recém-nascido (SBD, 2014-2015). Também é usado para identificar gestações de alto risco, onde consequências adversas podem ser evitadas e as intervenções são bastante simples (Coustan, 2012). Portanto, os novos critérios diagnósticos para o rastreamento de GDM são (IADPSG, 2010): Glicemia em jejum: 92 mg / dL; Após 1 hora: 180 mg / dL; Após 2 horas: 153 mg / d. 
O novo padrão internacional para o diagnóstico de DMG é baseado na correlação entre o índice de glicose no sangue materno e o peso ao nascer e a curva ultrapassa $90 \%$, pois os fetos gigantes são o maior indicador de hiperglicemia durante a gravidez, além dos fetos gigantes, obesidade e hiperinsulinemia fetal (Metzger et al., 2010; Lowe et al., 2012; WHO, 2013).

Os principais fatores de risco para DMG são: idade acima de 30 anos, baixa renda familiar, diagnóstico tardio de DMG, check-ups pré-natais tardios, sobrepeso ou obesidade, obesidade abdominal concentrada, parentes pela primeira vez com histórico familiar de diabetes, crescimento e desenvolvimento excessivos do feto, líquido amniótico excessivo, hipertensão induzida pela gravidez atual ou pré-eclâmpsia, história obstétrica de aborto espontâneo recorrente, malformações fetais, natimorto ou natimorto, bebês enormes, ovários policísticos e baixa estatura (Costa et al, 2015; SBD 2014-2015; Detsch et al., 2012). O DMG é uma patologia que pode vir a provocar diversas complicações maternofetais, sendo assim, éessencial que sejam feitos rastreios periódicamente para investigação de DM2 no pós parto (WHO, 2013).

O resultado trata-se de um estudo unicêntrico retrospectivo do tipo corte de dados referentes ao período de janeiro de 2015 a dezembro de 2018. O estudo foi realizado no Serviço de Terapia Intensiva adulto (materno) da Maternidade Ana Braga, inaugurada dia 10 de maio de 2004, que é uma unidade de saúde materno-fetal e neonatal de referência em parto de alto risco e em ensino (residência médica em ginecologia e obstetrícia), pertencente à rede pública estadual de saúde de Manaus Amazonas. Onde foi feito um levantamento das participantes, predominantemente puérperas, adultas jovens, com gestação em torno de 30 semanas, com pré-natal incompleto, que tiveram parto cesárea e procedentes. As pacientes adolescentes tinham idade entre 12 e 19 anos, enquanto que as adultas entre 20 e 45 anos. O estudo demonstrou que as mulheres adultas tiveram mais pré-eclâmpsia, mais comorbidades crônicas como hipertensão arterial, diabetes mellitus e obesidade (componentes da síndrome metabólica), além de iteratividade, anexectomias, abdome agudo hemorrágico e disfunções hemodinâmicas, enquanto que as adolescentes tiveram mais eclampsia, uso de medicamentos anti-hipertensivos, antiepilépticos e sulfato de magnésio, mais infecção de sítio cirúrgico, desnutrição, pneumotórax, além de apresentarem maior idade gestacional. (Pinheiro, 2019). Portanto as mulheres adultas apresentaram piores desfechos do que mulheres adolescentes admitidas a UTI em decorrência de complicações da gestação ou do parto causadas pelo DMG.

A importância de realizar corretamente o pré-natal e o rastreamento para o DMG está pode evitar complicações neonatais, tais como: aumento de partos cesáreos, Policitemia, icterícia, hipoglicemia e aumento do risco de mal formação congênita. A macrossomia é considerada a principal preocupação devido aos níveis elevados de glicose que o feto recebe da mãe, e a hipoglicemia pós-natal, em que a produção de insulina produzida pelo feto encontra-se aumentada para poder compensar a 73 quantidade de glicose que lhe era imposta (Ciências Saúde - 22 Sup 1:S93-S100, 2011).

A detecção do diabetes gestacional é altamente recomendada, devido à incidência de desfechos adversos maternofetais; pois, quando o diagnóstico é tardio ou a terapêutica não é seguida corretamente, aumentam os riscos de partos cesarianaS, pré-eclâmpsia, prematuridade, macrossomia, distocia de ombro, hipoglicemia neonatal, e morbimortalidade perinatal (Santos et al., 2012).

É importante que a gestante tenha conhecimento a respeito da DMG, para isso se faz necessário o acompanhamento a partir do pré-natal, buscando esclarecer dúvidas sobre a patologia, bem como orientar a gestante quanto à importância da terapia nutricional, visto que, hábitos alimentares saudáveis na pré e durante a gestação, contribuem para a redução de doenças associadas ao período gestacional e que associação de bons hábitos alimentares e a atividade física vão proporcionar grandes benefícios para a qualidade de vida e saúde da gestante (Ciências Saúde - 22 Sup 1:S93-S100, 2011).

\subsection{Terapia nutricional}

A importância da terapia nutricional nessas pacientes, com enfoque nos alimentos da região amazônica que ajudam à controlar e reduzir a glicemia sanguínea. Por isso temos alguns deles onde foram feitos estudos com compostos bióativos 
dessas frutas. Alguns deles são: Tucumã é uma fruta da Amazônia que vem sendo utilizada para ajudar a prevenir e a tratar a diabetes, pois ela é rica em ômega-3, gordura que diminui a inflamação e o colesterol alto, ajudando também no controle do nível de açúcar no sangue, Graviola cultivada na região norte, suas fibras ajudam a reduzir os níveis de glicose do sangue. Com isso, a fruta e o suco podem ser considerados aliados das pessoas que sofrem com diabetes, Cupuaçu melhora o equilíbrio da glicose no organismo, Guaraná pode ajudar no controle da diabete tipo 2. Testes in vitro indicam que algumas substâncias bioativas do guaraná poderiam agir no controle glicêmico (Chaves,2016).

\subsubsection{O tucumã}

O tucumã (Astrocaryum aculeatum) é um fruto presente na região amazônica, muito consumido in natura e/ou subprodutos, como, por exemplo, recheios de sanduíches, cremes e sorvetes, devido ao seu potencial nutritivo (Carneiro et al., 2017; Santos et al., 2018). É indicado para alimentação humana e de animais domésticos, uma vez que o mesocarpo se caracteriza por uma fonte alimentícia altamente calórica, já que tem elevado conteúdo de lipídeos. Possui quantidades significativas do precursor da vitamina A (Yuyama et al., 2008), teores de fibras e vitamina E (Ribeiro \& Soares, 1995), além de ação antioxidante (Vieira et al., 2017).

\subsubsection{Cupuaçu}

O cupuaçu é uma fruta ácida, de sabor exótico e agradável, rico em sais minerais (potássio, fósforo, magnésio, ferro e zinco), vitamina C (102 mg/100 g), compostos fenólicos (3,5 a 4,9 mg de equivalente em catequina/g amostra seca), com elevada atividade antioxidante (1,7 a 2,0 $\mu \mathrm{M}$ de Trolox/g). Além disso, o cupuaçu apresenta alto teor de pectina, comparável ao da maçã, fibra alimentar solúvel que, segundo alguns trabalhos, têm demonstrado redução dos níveis séricos de colesterol e triglicerídeos em ratos e humanos (Martins, 2008; Pereira, Abreu \& Rodrigues, 2018).

\subsubsection{Graviola}

Considerada um fitoterápico natural desde muito tempo pelos povos indígenas, a graviola vem se destacando por ser rico em bio ativos atuantes contra uma ampla gama de doenças humanas, entre elas o câncer. Esses compostos bi ativos são encontrados em partes que não são usadas geralmente e tornam-se residuais como, casca, raiz, sementes e folhas (Freitas, Moraes \& Silva, 2017). Além das propriedades anticancerígenas, o fruto também contém níveis satisfatórios de vitamina C, que pode trazer diversos benefícios como a atuação antioxidante que protege as células em geral, além do alto teor de fibras, importante para o bom funcionamento do sistema digestório. O fruto pode ser utilizado ainda ajudando no tratamento de abcessos, câncer, insônia, diabetes, hipertensão, depressão, reumatismo e outras doenças (Ramos et al., 2015).

\subsubsection{Guaraná}

O guaraná (Paullinia cupana) por ser um exclusivamente brasileiro e com valor altamente energético, sendo utilizado frequentemente em suplementos energéticos por conter substâncias estimulantes que agem contra o desgaste físico e mental (Ribeiro, Coelho \& Barreto, 2012). Dentre suas substâncias, encontram-se dois principais constituintes químicos do guaraná: as metis xantinas e a cafeína. A suplementação nutricional utilizando o guaraná como um dos recursos ergo gênicos consiste na melhoria da performance dos atletas, consumindo-o antes do exercício (Silveira, Amorim \& Burian, 2018).

$\mathrm{O}$ tratamento da hiperglicemia provou-se um fator fundamental na contenção dos danos causados pelo DMG, como ilustrado pela fisiopatologia da doença, sendo assim o principal foco da terapia. A manutenção do tripé das necessidades da mãe, do feto e da capacidade da mãe em prover e manter as necessidades do feto depende de uma regulação que, mesmo quando desbalanceada, pode ser reconduzida à normalidade por estratégias terapêuticas, devolvendo a paciente a um estado 
hígido. A prevenção de doenças crônicas não transmissíveis é um pilar que deve ser fortalecido na sociedade atual, por sua prevalência crescente, e a DMG, que é um prólogo de doenças que se manifestam na sequência, é uma patologia que não pode ser esquecida durante o acompanhamento pré-natal. (Araujo Muniz et al., 2014).

A intervenção nutricional é uma importante aliada no controle do Diabete Mellitus Gestacional, trazendo benefícios à saúde materno-fetal. É fato o reconhecimento de que as necessidades nutricionais durante o período gestacional e na lactação são similares às das mulheres sem diabetes. A terapia nutricional para DMG deve ser direcionada para escolhas alimentares que garantam um apropriado ganho de peso e controle glicêmico. (Carvalho Padilha et al., 2010)

Diante disso é evidente que a intervenção nutricional é uma importante aliada no controle do Diabetes Mellitus Gestacional, pois esta trás diversos benefícios à saúde tanto da mãe como do feto. Assim, a terapia nutricional para DMG deve enfatizar escolhas alimentares que assegure um ganho de peso apropriado, controle glicêmico e ausência de corpos cetônicos. Portanto, a participação do nutricionista se torna imprescindível na atenção básica, principalmente na orientação de medidas que busque a redução dos agravos de saúde provenientes dos maus hábitos alimentares, visto que o aumento de doenças metabólicas vem se tornando a cada dia mais comum, quanto maior for o nível de conhecimento das gestantes melhor será a compreensão da mesma a respeito da DMG. (Carvalho Padilha et al., 2010)

\section{Considerações Finais}

O presente estudo sobre o DMG, possibilitou um levantamento global sobre esse distúrbio metabólico que afeta 1 à $14 \%$ da população brasileira. Onde o enfoque desse estudo foi na cidade de Manaus no estado do Amazonas. Assunto de suma importância para a saúde pública, que traz grandes complicações durante o período gestacional e até mesmo após ele. O conhecimento científico do tema, a realização correta do pré-natal, como o rastreamento metabólico para diagnóstico precoce do DMG, com o intuito de evitar, tratar e controlar esse grave distúrbio que vem sendo cada vez frequente e preocupante na vida das pessoas, por acarretar diversos problemas à saúde. Destacamos alguns da região Amazonica que ajudam no tratamento da DMG. Portanto é imprescindível o acompanhamento no pré-natal, junto com a terapia nutricional adequada e individualizada, orientando sempre a gestante sobre o hábito alimentar saudável e atividade física, afim de evitar e controlar o Diabete.

\section{Referências}

Amanda, K. D. M., Uiara, M. F. L., Maria, F. N. N., Lucineide, S. A., Laysa, M. O. N. \& Maria, M. N. (2018). O Papel Da Agricultura Familiar No Programa Nacional De Alimentação Escolar: Uma Revisão Literária, João Pessoa.

Ana Maria, C. M., Marcia, F. W., Erica, L. A., \& Claudia, M. B. (2013). O Papel da Alimentação escolar na formação dos hábitos alimentares. Ver. Paul. Pediatr., São Paulo, 31(3), 324- 330.

Almeida, A. L. (2012). Educação e saúde: hábito alimentar de crianças referentes á alimentação escolar. Monografia.

Alessandra, P. J. (2016). Alimentação Saudável na Escola: Promovendo A Saúde e Construindo Ações de Segurança Alimentar, TCC, Belo Horizonte - Minas Gerais.

Brasil. (2021). Ministério de Educação. Programa de Suplementação: Sobre o PNAE. 2021.

Brasil, Aquisição de Produtos da Agricultura Familiar para o Programa Nacional de Alimentação Escolar Acesso em 15/05/2021 as 13:30 h.

Brasil. (2017). Boas práticas de agricultura familiar para a alimentação escolar/Programa nacional de Alimentação escolar. - Brasília: FNDE.

Brasil. (2015). Ministério da Educação, Cartilha Nacional da Alimentação Escolar, Ministério da Educação, Brasília, DF, (2a ed.).

Brasil. (2013). Ministério da Saúde, Política Nacional de Alimentação e Nutrição Brasília - DF.

Brasil. (2012). Ministério do Desenvolvimento Social e Combate à Fome. Secretaria Nacional de Segurança Alimentar e Nutricional. Marco de Referência de educação alimentar e nutricional para as políticas públicas. Brasília: Ministério do Desenvolvimento Social e Combate à Fome. 68. 
Brasil. (2014). Ministério da Educação, Cartilha, Orgânicos na alimentação Escolar a Agricultura Familiar alimentado o saber.

Brasil. (2018). Ministério da Educação, Fundo Nacional de Desenvolvimento da Educação, Manual de apoio para as atividades técnicas do Nutricionista do Âmbito do PNAE, Brasília - DF. 60.

Brasil. Ministério da Educação, Programa Nacional de Alimentação Escolar Cardápios na Alimentação Escolar Coordenação de Segurança Alimentar e Nutricional - COSAN.

Brasil. Ministério de Educação. Programa de Suplementação: Alimentação e Nutrição < https://www.fnde.gov.br/index.php/programas/pnae/pnae-eixos-deatuacao/pnae-alimentacaoe-nutricao>.

Barbosa, N. V. S., Machado, N. M. V., Soares, M. C. V., \& Pinto, A. R. R. (2013). Alimentação na escola e autonomia - desafios e possibilidades. Cien Saude Colet, 18(4), 937-945.

Bizzo, M. L. G., \& Leder, L. (2005). Educação nutricional nos parâmetros curriculares nacionais para o ensino fundamental. Rev Nutr. 18(5), 661-667.

Burity, V., Franceschini, T., Valente, F., Recine, E., Leão, M., \& Carvalho, M. F. (2010). Direito Humano à Alimentação Adequada no Contexto da Segurança Alimentar e Nutricional. Brasília.

Caniné, E. S., \& Ribeiro, V. M. B. (2007). A prática do nutricionista em escolas municipais do Rio de Janeiro: um espaço tempo-educativo. Ciênc Educ. 13(1), 47-70.

Camozzi, A. B. et al. (2015). O Promoção da Alimentação Saudável na Escola: realidade ou utopia? Cad. Saúde Colet., 23 (1), $32-7$.

Costa, E. Q., Ribeiro, V. B., \& Ribeiro, E. C. O. (2011). Programa de alimentação escolar: espaço de aprendizagem e produção de conhecimento. Rev Nutr. 14(3), 225-229.

Danelon, M. A. S., Danelon, M. S., \& Silva, M. V. (2016). Serviços de alimentação destinados ao público escolar: análise da convivência do Programa de Alimentação Escolar e das cantinas. Segurança Alimentar e Nutricional. v. 13 Campinas: Unicamp, 85-94.

Davanço, G. M., Taddei, J. A. A. C., Gaglianone, C. P. (2004). Conhecimentos, atitudes e práticas de professores de ciclo básico, expostos e não expostos a curso de educação nutricional. Rev Nutr, 17(2), 177-184.

Fiore, E. G., Jobstraibizer, G. A., Silva, C. S., \& Cervato-Mancuso, A. M. (2012). Abordagem dos temas alimentação e nutrição no material didático do ensino fundamental: interface com segurança alimentar e nutricional e parâmetros curriculares nacionais. Saúde, 21(4), 1063-1074.

Fonseca, A. N., \& Gomes, J. C. (2015). Merenda Escolar: um Estudo Exploratório sobre a Implementação do Programa Nacional Alimentação na Escola PNAE, na Unidade Integrada Padre Newton Pereira em São Luís, EDUCARE, PUC.

FNDE, Agencia Nacional de Vigilância Sanitária. (2013). Guia de Instruções das Ferramentas para as Boas Práticas na Alimentação Escolar, Brasília.

Flávio, E. F., Barcelos, M. F. P., \& Lima, A. L. (2004). Avaliação química e aceitação da merenda escolar de uma escola estadual de Lavras-MG. Cienc agrotec, 28(4):840-847

Guia Prático. (2020). Alimentação escolar indígena e de comunidades tradicionais, PNAE Indígena no Amazonas, Brasília.

Hernandez, A. B., Slavutzky, S. M. B., \& Padilha, D. M. P. (2008). Avaliação do consumo da merenda escolar em escolas municipais de Porto Alegre. Rev Fac Odonto. Porto Alegre. 49(1), 26-30.

Jones, A. M., \& Zidenberg-Cherr, S. (2015). Exploring nutrition education resources and barriers, and nutrition knowledge in teachers in California. $J$ Nutr Educ Behav. 47(2), 162-169.

Lima, Ana Patrícia Silva Carvalho. (2016). Importância da Alimentação para Melhorias na Aprendizagem de Crianças em Unidades Públicas De Ensino. Revista Somma, Teresina, 2(2), 74-83.

Marlette, M. A., Templeton, S. B., \& Panemangalore, M. (2005). Food Type, Food Preparation, and Competitive Food Purchases Impact School Lunch Plate Waste by Sixth-Grade Students. J Am Diet Assoc, 105(11), 1779-1782.

Marconi, M. A., \& Lakatos, E. M. (2010). Fundamentos de metodologia científica. Editora Atlas.

Mello, A. L., Vidal Junior, P. O., Sampaio, L. R., Santos, L. A. S, Freitas, M. C. S, \& Fontes, G. A. V. (2012). Perfil do nutricionista do Programa Nacional de Alimentação Escolar na região Nordeste do Brasil. Rev Nutr, 25(1), 119-132.

Muller. (2011). Boas Práticas de Manipulação de Alimentos com Merendeiras. São Miguel do Oeste, Uniedu.

Muniz, V. M., \& Carvalho, A. T. (2017). O Programa Nacional de Alimentação Escolar em município do estado da Paraíba: um estudo sob o olhar dos beneficiários do Programa. Revista de Nutrição, 20(3) Campinas.

Neto, L. G., Bezerra, J. A. B., \& Santos, A. N. (2012). Qualidade na merenda escolar: intervenções gastronômicas. Encontro Nacional de Didática e Práticas de Ensino Campinas: UNICAMP.

Oliveira, R. V. (2016). O Programa Nacional De Alimentação Escolar: Uma Análise De Sua Implementação Nas Escolas Da Rede Estadual De Ensino De Manaus, Juiz De Fora.

Perroni, C. (2013). Boa alimentação interfere na função cerebral e aumenta a concentração. 
Research, Society and Development, v. 10, n. 15, e14101522632, 2021

(CC BY 4.0) | ISSN 2525-3409 | DOI: http://dx.doi.org/10.33448/rsd-v10i15.22632

Perovano, D. G. (2014). Manual de Metodologia Científica. Editora Juruá.

Proença, R. P. C. (2017). Alimentação e globalização: algumas reflexões, Cienc. Cult. 62(4).

Ribeiro, G. N. M., \& Silva, J. B. L. (2013). Alimentação no Processo de aprendizagem. Revista Eventos Pedagógicos, Mato Grosso, 4(2), 77-85.

Ribeiro, Ana L. P., Silene C., \& Djulia T. B. (2013). Programa Nacional de Alimentação Escolar (PNAE) e a participação da agricultura familiar em municípios do Rio Grande do Sul, Revista Gestão e Desenvolvimento em Contexto- Gedecon, 1(01), 36-49.

Rodrigues, L. P. F., Zaneti, I. C. B., \& Laranjeira, N. P. (2012). Sustentabilidade, segurança alimentar e gestão ambiental para a promoção da Saúde e qualidade de vida.

Schneider, S. (2003). Teoria social, agricultura familiar e pluriatividade. Revista Brasileira de Ciências Sociais, 18(51), 99-121.

Santos, L. A. S. (2012). O fazer educação alimentar e nutricional: algumas contribuições para reflexão. Cien Saude Colet. 17(2), 453-462.

SEDUC, Secretaria de Educação do Estado do Amazonas. Programa de Regionalização da Merenda Escolar (Preme), < http://www.educacao.am.gov.br/programa-de-regionalizacao-damerenda-escolar-preme/>.

Santos, I. H. V. S., Ximenes, R. M., \& Prado, D. F. (2018). (2018). Avaliação do cardápio e da aceitabilidade da merenda oferecida em uma escola estadual de ensino fundamental de Porto Velho, Rondônia - Saber Científico, Porto Velho.

Santos, D. M. (2017). TCC - a Alimentação Escolar como Estratégia de Educação Alimentar e Nutricional: Uma Revisão da Literatura, Vitória de Santo Antão.

Santori, A., \& Amancio, R. D. (2012). Pescado: importância nutricional e consumo no Brasil. Segurança Alimentar e Nutricional, Campinas, 19(2), 83-93.

Sampaio, H. A. C., Sabry, M. O. D., Rêgo, J. M. C., Passamai, M. P. B., Sá, M. L. B., Matos, M. R. T., \& Passos, T. U. (2017). Estado nutricional de escolares de um bairro da periferia da cidade de Fortaleza - Ceará. Revista Nutrição em Pauta, (84).

Vieira, A. V., Corso, A. C. T., \& González-Chica, D. A. (2014). Organic food-related educational actions developed by dieticians in Brazilian municipal schools. Rev Nutr. 27(5):525-535.

Watts, S. O., Piñero, D. J, Alter, M. M., \& Lancaster, K. J. (2012). An Assessment of nutrition education in selected counties in New York State elementary schools. J Nutr Educ Behav. 44(6):715-725. 